\title{
Overcoming gas ionization limits with divided-pulse nonlinear compression
}

\section{G. W. Jenkins, C. Feng, J. Bromage}

Laboratory for Laser Energetics, University of Rochester, 250 East River Road, Rochester, NY 14623-1299, USA

Gas-filled hollow-core fibers (HCFs) have been successfully used for spectrally broadening high-averagepower $\mathrm{Yb}$ systems to $\sim 10$-fs pulse durations for a number of applications. However, pulse-energy scaling in an HCF is limited by self-focusing and ionization [1]. Our simulations show that divided-pulse nonlinear compression (DPNLC), shown schematically in Fig. 1(a), can overcome those limits and improve the spectral broadening of high-energy pulses.

The self-focusing in an HCF can be controlled by reducing the nonlinear index of the gas as the peak power of the pulse is increased [2,3]. The gas pressure $(p)$ is a convenient variable to tune the nonlinear index and should be set below Tempea and Brabec's threshold of 9\% peak power in the second-order mode. In our simulations, we set the gas pressure to half this value and vary the fiber radius to vary the strength of plasma effects. We simulate a 10-mJ, 1-ps (FWHM) pulse centered at a 1030-nm wavelength, propagating through the HCF with a model adapted from Horak and Poletti [4]. Figure 1 shows that the onset of ionization at a fiber diameter of about 550 $\mu \mathrm{m}$ leads to significant energy losses in the fiber and a reduction in the peak power achievable.

(a)

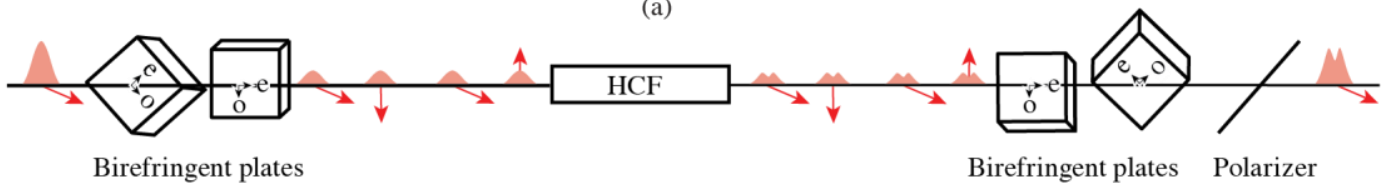

(b)

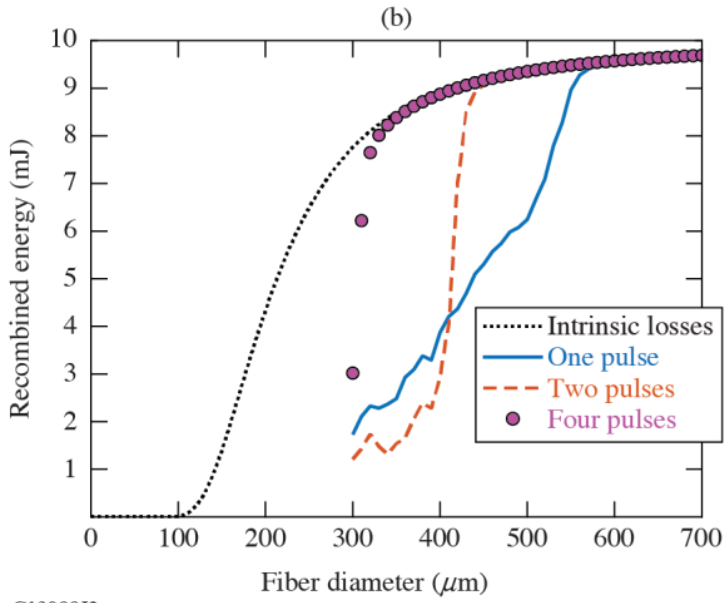

(c)

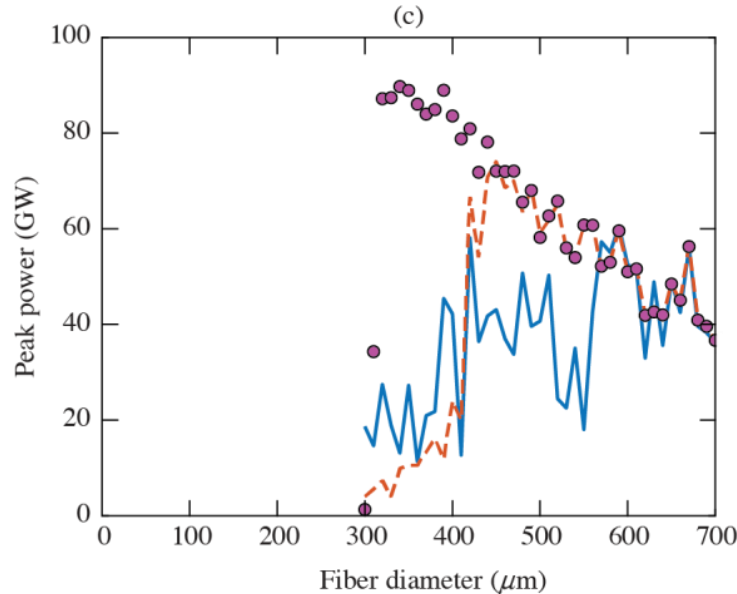

Fig. 1 (a) Schematic of DPNLC. (b) Simulated output energy after HCF and divided pulse recombination stages for a range of fiber diameters. Onset of ionization is clearly visible around $550 \mu \mathrm{m}, 400 \mu \mathrm{m}$, and $300 \mu \mathrm{m}$ for one, two, and four pulses, respectively, and results in large energy losses. (c) Simulated peak power after recombination and compression with up to second-order phase.

In DPNLC, a high-energy pulse is divided into multiple low-energy pulses, which are spectrally broadened in the fiber (note that the gas pressure is re-optimized for the low-energy pulses), and then they are recombined to form a high-energy, spectrally broadened pulse [5]. Figure 1 displays our simulations of the recombined pulse parameters if two 5-mJ, or four 2.5-mJ pulses are spectrally broadened instead of one 10-mJ pulse. The ionization limit is shifted to smaller fiber diameters, leading to higher energy throughputs, larger spectral broadening factors, and higher peak powers after the pulses are recombined.

This material is based upon work supported by the Department of Energy National Nuclear Security Administration under Award Number DE-NA0003856, the University of Rochester, and the New York State Energy Research and Development Authority. The support of DOE does not constitute an endorsement by DOE of the views expressed in this abstract.

\section{References}

[1] C. Vozzi, M. Nisoli, G. Sansone, S. Stagira, and S. De Silvestri, "Optimal Spectral Broadening in Hollow-Fiber Compressor Systems," Appl. Phys. B 80, 285 (2005).

[2] G. Tempea and T. Brabec, "Theory of Self-Focusing in a Hollow Waveguide," Opt. Lett. 23, 762 (1998).

[3] N. Milosevic, G. Tempea, and T. Brabec, “Optical Pulse Compression: Bulk Media Versus Hollow Waveguides,” Opt. Lett. 25, 672 (2000).

[4] P. Horak and F. Poletti, "Multimode Nonlinear Fibre Optics: Theory and Applications," in Recent Progress in Optical Fiber Research, edited by M. Yasin (InTechOpen, Rijeka, Croatia, 2012), pp. 1-24.

[5] H. Jacqmin et al., "Passive coherent combining of CEP-stable few-cycle pulses from a temporally divided hollow fiber compressor,"

Optics Letters, vol. 40, no. 5, pp. 709-712, Mar 2015. 